\title{
Chronic Lymphocytic Leukemia in Remission
}

National Cancer Institute

\section{Source}

National Cancer Institute. Chronic Lymphocytic Leukemia in Remission. NCI Thesaurus.

Code C3587.

Chronic lymphocytic leukemia not growing, responding to treatment. 